\title{
Ziele des WRFU
}

$\mathrm{Zu}$ den obersten Zielen des WRFU gehört erstens die Erfassung und Förderung aller natürlichen Ressourcen der Erde, die ökonomisch nutzbar und unter den Nationen verhandelbar sind. Zweitens sind die Daten laufend zu analysieren, zu quantifizieren und entsprechende Massnahmenpläne vorzuschlagen. Drittens müssen in den Wahlverfahren Wege und Strategien umgesetzt werden, damit Entscheide in einem (noch zu definierenden System) verbindlich unter allen Völkern verteilt werden. Eine weitere primäre Aufgabe des WRFU wird, das Erfassen und Umverteilen von Rohstoffen und Energiequellen sowie das Bearbeiten der Endlager toxischer Abfälle sein.

Der Weltrat ist ermächtigt, nachhaltigen und globalen Umweltschutz zu leisten. Er bewältigt eine zentrierte Klimaarbeit, die weltweit greift und überall rechtlich anerkannt ist.

Über die Umverteilung und Zuteilung aller natürlichen Ressourcen (Luft, Sonne, Wasser) und der fossilen Energieträger (Öl, Kohle etc.) sowie über die Frage, wie wir weltweit toxische Abfälle entsorgen, wird das globale Parlament für Umweltschutz (WRFU) entscheiden.

Naturkräfte sind, wie wir aus der Wissenschaft wissen, voneinander und untereinander durch ihre eigenen an das Universum gebundenen Gesetze abhängig. Deshalb sind sie nicht für einzelne Regierungen verhandelbar. Hingegen benötigen sie den Schutz und die unermüdliche Pflege einer internationalen Weltgemeinschaft. 